\title{
Simulation process of the heat transfer in multilayered structures
}

\author{
Yuriy Starodub ${ }^{1}$, Roman Veselivskyy ${ }^{1, *}$ and Oleksandr Vasylenko ${ }^{2}$ \\ ${ }^{1}$ Lviv State University of Life Safety, 79000, 35 Kleparivska St., Ukraine \\ ${ }^{2}$ LLC "Scientific-Production Enterprise ADR Expert", 79000, 2 Gen. Kurmanovycha St., Ukraine
}

\begin{abstract}
The mathematical model of heat transfer in a metal- expanded polystyrene- metal system is considered in the paper, taking into account the stratification and specificity of heat sources that appear in the process of thermal destruction of expanded polystyrene. Specificity of heat sources is based on the transition of expanded polystyrene from solid to gaseous state during the heating of the prototype in the furnace behind the temperature regime of a standard fire. Considered the problem of the process of nonstationary heat conduction, the simulation was performed taking into account the transition time of expanded polystyrene into the gaseous phase, and also taking into account the time of action of additional heat sources using a numerical scheme based on the finite difference method modelling. The time of the loss of the heat-insulating capacity of the three-layered wall system was determined. A comparative analysis of the results of theoretical and experimental studies to assess their fire resistance was carried out.
\end{abstract}

\section{Introduction}

The urgency of saving energy resources today is an important task of the construction industry. In this regard, new methods of energy saving are developed, aimed at the rational use of economic resources. This concerns the initial stages of designing buildings and structures for various purposes, as well as the final stage of their construction.

For one of these technologies include the production of multilayer walling, in which they aim to combine the appropriate level of heat and sound insulation, fire resistance, hygiene requirements and the mechanical strength at the lowest cost, while ensuring optimal installation and construction conditions. Such multi-layered enclosing structures consist of a frame and fillers. The frame is made of profiled metal, OSB (Oriented Strand Board) and QSB (Qualitative Strange Board - high-quality and high-strength wood chipboard) plates, also known for the use of magnesite boards. As filler polystyrene (hereinafter - PS), mineral wool, polyurethane foam is used.

\footnotetext{
* Corresponding author: roman_veselivskuy@yahoo.com
} 


\section{Analysis of recent research and publications}

Since experimental studies of fire resistance of such enclosing structures are quite lengthy and require significant financial costs, the actual task is to construct mathematical models of such processes in order to reduce the costs of these studies and extend the results to other materials and multi-layer structures.

The process of heating of the system under consideration is accompanied by complex interconnected processes of heat transfer between the PS and the surrounding medium, chemical and physical transformations in the investigated temperature range, including chemical destruction of polymers, their melting, and possibly combustion with loss of mass, changes in the density and physical structure of materials $[1,2,3]$.

Attention should also be paid to a wide range of numerical values of thermophysical characteristics of materials in a multilayer structure, according to which materials are divided into heat conducting and heat-insulating materials $[4,5]$. All these materials are present in the investigated design simultaneously, which significantly complicates the construction of the model and the obtaining of numerical results.

For a theoretical study of heat transfer in a system, it is necessary to use a mathematical model, taking into account the layering of the system and the specifics of heat sources.

The resulting mathematical model should provide a simple means of assessing the fire resistance of the system and be as simple as possible for practical use.

Consideration of the specific geometrical dimensions of the system, large areas of the walls at their relatively small thickness, the assumption is, of their relatively uniform heating. In a fire on one side along the areas length and width, as well as their complexity of physical and chemical processes that occur inside the insulation, the transience processes are modelled with the use of the mathematical model of non-stationary thermal conductivity. Consideration of the processes depend on one-dimensional coordinate system layering and a possible dependence of temperature, heat flux, etc. materials characteristics of corresponding layers.

It is necessary to solve the system of equations describing such a model by numerical method, since it is inexpedient to change the solution method when improving the model.

The aim of the study is to determine the time of loss of the thermal insulation capacity of a three-layer wall system consisting of two layers of profiled steel $0.5 \mathrm{~mm}$ thick and a layer of PS $100 \mathrm{~mm}$ thick.

\section{Formulation of the study method}

\subsection{Statement of the problem of thermal conductivity for the metal-PS-metal system}

We consider the one-dimensional heat conduction problem for a three-layer plate, which we simulate an actually existing multilayer panel consisting of metal-PS metal (Fig. 1). 


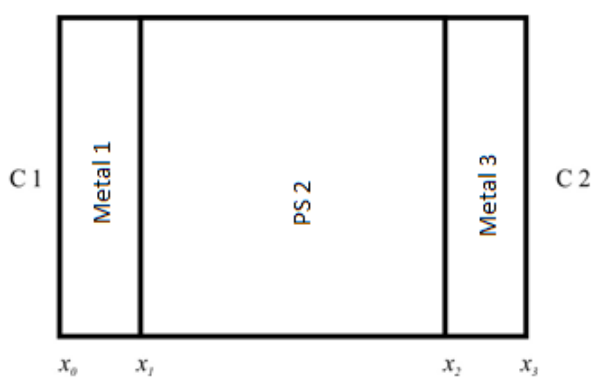

Fig. 1. Schematic representation of the metal-PS -metal system. Metal 1, PS 2, Metal 1 - materials of the medium, C1, C2 - external media, x0, x1, x2, x3 - coordinates of the layers system.

We consider that in the PS when a certain critical temperature is reached, chemical reactions and destruction begin to occur with the release of heat, that is, the process takes place in two stages.

The first stage lasts until the time moment $\tau=\tau_{\mathrm{cr}}$, when the temperature $T_{2}$ in the PS reaches a critical value $T_{\mathrm{cr}}$.

The heat conduction equations and the initial conditions have the form $[4,6]$ :

$$
\begin{gathered}
\frac{\partial T_{i}}{\partial \tau}=a_{i} \frac{\partial^{2} T_{i}}{\partial x^{2}} ; \\
\mathrm{T}_{i \mid \tau=0}=T_{0}, \quad i=1,2,3 .
\end{gathered}
$$

On the limiting surfaces of the body-medium heat exchange takes place according to Newton's law:

$$
\begin{aligned}
& \lambda_{1} \frac{\partial T_{1}}{\partial x}=-\alpha_{1}\left(T_{C 1}(\tau)-T_{1}\right), \text { where } x=x_{0}=0 ; \\
& \lambda_{3} \frac{\partial T_{3}}{\partial x}=\alpha_{2}\left(T_{C 2}(\tau)-T_{3}\right), \text { where } x=x_{3}
\end{aligned}
$$

Conditions for an ideal thermal contact were created on the interface surfaces of the system:

$$
T_{i}=T_{i+1}, \quad \lambda_{i} \frac{\partial T_{i}}{\partial x}=\lambda_{i+1} \frac{\partial T_{i+1}}{\partial x} \text { where } \mathrm{x}=\mathrm{x}_{i},
$$

Here $T_{i}$ - temperature of the $\mathrm{i}$ - th layer of the system, $T_{C 1}, T_{C 2}$ - the temperature of the media in which the fire occurs and from the opposite side, respectively; $a_{i}=\lambda_{i} / \rho_{i} \omega_{i}$, and $\lambda_{i}, \omega_{i}$ coefficients of thermal diffusivity, thermal conductivity and specific heat; $\rho_{i}$ - density; $\alpha_{l}$ - heat transfer coefficient from the metal surface $\left(l=1\right.$ at $x=x_{0}, l=2$ at $\left.x=x_{3}\right)$.

At the second stage for time $\tau>\tau_{\text {cr }}$ we assume instantaneous conversion of PS into a smoke- gas-air mixture for which the right side of the heat equation includes a heat source. Such an assumption reduces the estimated fire resistance time and is therefore acceptable.

That is, for $\tau>\tau_{\text {cr }}$ in region 2 of the heat equation will be written: 


$$
\begin{aligned}
& \frac{\partial T_{2}}{\partial \tau}=a_{2}^{g} \frac{\partial^{2} T_{2}}{\partial x^{2}}, \quad \text { where } T_{2}<T_{\mathrm{cr}} \\
& \frac{\partial T_{2}}{\partial \tau}=a_{2}^{g} \frac{\partial^{2} T_{2}}{\partial x^{2}}+q, \text { where } T_{2} \geq T_{\mathrm{cr}}
\end{aligned}
$$

Accordingly, the conditions of contact (5) have the form:

$$
\begin{aligned}
& T_{1}=T_{2}, \quad \lambda_{1} \frac{\partial T_{1}}{\partial x}=\lambda_{2}^{g} \frac{\partial T_{2}}{\partial x}, \text { where } x=x_{1}, \\
& T_{2}=T_{3}, \quad \lambda_{2}^{g} \frac{\partial T_{1}}{\partial x}=\lambda_{3} \frac{\partial T_{3}}{\partial x}, \quad \text { where } x=x_{2} .
\end{aligned}
$$

Here $q$ - power of thermal sources, $a_{2}^{g}=\lambda_{2}^{g} / \rho_{2}^{g} \omega_{2}^{g}$, where $g$ the index refers to values related to the smoke / gas / air mixture. We assume that thermal sources operate throughout the $\tau_{q}$ time.

To determine the source $q$ in the heat conduction equation, which characterizes the intensity of energy sources that are nonlinear with respect to temperature and is directly related to the rate of chemical reactions, it is necessary to model the kinetics of chemical reactions that occur in the polymer quite accurately. The rate of these reactions is significantly influenced by heat fluxes and temperature. The quantitative calculation of the effect of heat fluxes and temperature on the rate of chemical reactions in polymers is not completely solved by the scientific problem $[1,2,7,8,9]$. Therefore, in this paper we use a simplified empirical model that takes into account only the power and time of action of thermal sources when a certain critical temperature is reached.

\subsection{Numerical scheme for solving the heat conduction problem for a metal- PS -metal system}

The construction of a numerical scheme based on the finite difference method $[10,11]$ is carried out as follows. We believe that the solution of the problem exists, unique and sufficiently smooth for approximation. The region of continuous variation of the coordinate along the thickness of the plate $\mathrm{x}$ and time replace by a discrete set of nodes, and instead of functions continuous arguments $\mathrm{x}$ and consider the functions discrete arguments that are defined in the grid nodes by coordinate and time. The grid breakdown over the spatial variable has the form:

$$
x_{i j}=x_{i-1}+(j-1) h_{i}, i=1,2,3 ; j=1, \ldots N_{i} ; \ldots h_{i}=\frac{x_{i}-x_{i-1}}{N_{i}-1},
$$

and breakdown by time: $\tau_{m}=m \Delta \tau, m=0,1,2, \ldots$.

Here $N_{i}$ - the number of nodes in the i-th layer, $h_{i}$ - the grid step in the $i$ th layer, $\Delta \tau$ - step by time.

We are looking for a grid function $T_{i, j}^{m}$, defined by the formula: 


$$
T_{i, j}^{m}=\left\{\begin{array}{lll}
T\left(x_{i j}, \tau_{m}\right), & i=1,2,3 & j=2, \ldots, N_{i}-1 \\
\lim _{x \rightarrow x_{i}-0} T\left(x, \tau_{m}\right), & i=1,2,3 & j=N_{i} ; \\
\lim _{x \rightarrow x_{i-1}+0} T\left(x_{i j}, \tau_{m}\right), & i=1,2,3 & j=1 .
\end{array}\right.
$$

which at $\tau \leq \tau_{\text {cr }}$ satisfies the systems of finite-difference relations:

$$
\begin{gathered}
\frac{T_{i, j}^{m+1}-T_{i, j}^{m}}{\Delta \tau}=a_{i} \frac{T_{i, j+1}^{m}-2 T_{i, j}^{m}+T_{i, j-1}^{m}}{h_{i}^{2}}, i=1,2,3, j=2, \ldots N_{i}-1, m=0,1, \ldots, m_{\mathrm{cr}}-1 \\
\lambda_{i} \frac{3 T_{i, N_{i}}^{m+1}-4 T_{i, N_{i}-1}^{m+1}+T_{i, N_{i}-2}^{m+1}}{2 h_{i}}=\lambda_{i+1} \frac{-3 T_{i+1,1}^{m+1}+4 T_{i+1,2}^{m+1}-T_{i+1,3}^{m+1}}{2 h_{i+1}}, i=1,2, m=0,1, \ldots, m_{\mathrm{cr}}-1 ;(1) \\
T_{i, N_{i}}^{m+1}=T_{i+1,1}^{m+1}, i=1,2, m=0,1, \ldots, m_{\mathrm{cr}}-1 \\
\quad m=0,1, \ldots, m_{\mathrm{cr}}-1 ; \\
\lambda_{1} \frac{-3 T_{1,1}^{m+1}+4 T_{1,2}^{m+1}-T_{1,3}^{m+1}}{2 h_{1}}=-\alpha_{1}\left(t_{\tilde{N} 1}\left(\tau_{m+1}\right)-T_{1,1}^{m+1}\right), m=0,1, \ldots, m_{\mathrm{cr}}-1 \\
T_{i, j}^{0}=T_{0}, i=1,2,3, j=1, \ldots N_{i}
\end{gathered}
$$

When $\tau>\tau_{\mathrm{cr}}$ instead of equations (9), the following equations will be satisfied:

$$
\begin{gathered}
\frac{T_{2, j}^{m+1}-T_{2, j}^{m}}{\Delta \tau}=a_{2}^{g} \frac{T_{2, j+1}^{m}-2 T_{2, j}^{m}+T_{2, j-1}^{m}}{h_{2}^{2}}+q, j=2, \ldots j_{\mathrm{cr}}, \ldots, m=m_{\mathrm{cr}}, m_{\mathrm{cr}}+1, . ., m_{\mathrm{cr}}+n_{q_{\tau}} ;(15.1) \\
\frac{T_{2, j}^{m+1}-T_{2, j}^{m}}{\Delta \tau}=a_{2}^{g} \frac{T_{2, j+1}^{m}-2 T_{2, j}^{m}+T_{2, j-1}^{m}}{h_{2}^{2}}, j=2, \ldots j_{\mathrm{cr}}, m=m_{\mathrm{cr}}+n_{q_{\tau}}, m_{\mathrm{cr}}+n_{q_{\tau}}+1, \ldots \\
j=j_{\mathrm{cr}}+1, \ldots, N_{2}-1 ; m=m_{\mathrm{cr}}, m_{\mathrm{cr}}+1, m_{\mathrm{cr}}+2, \ldots
\end{gathered}
$$

and instead of equations (10) - the equation:

$$
\begin{aligned}
& \lambda_{1} \frac{3 T_{1, N_{1}}^{m+1}-4 T_{1, N_{1}-1}^{m+1}+T_{1, N_{1}-2}^{m+1}}{2 h_{1}}=\lambda_{2}^{g} \frac{-3 T_{2,1}^{m+1}+4 T_{2,2}^{m+1}-T_{2,3}^{m+1}}{2 h_{2}}, \\
& m=m_{\mathrm{cr}}, m_{\mathrm{cr}}+1, m_{\mathrm{cr}}+2, \ldots \\
& \lambda_{2}^{\gamma} \frac{3 \mathrm{~T}_{2, \mathrm{~N}_{2}}^{\mu+1}-4 \mathrm{~T}_{2, \mathrm{~N}_{2}-1}^{\mu+1}+\mathrm{T}_{2, \mathrm{~N}_{2}-2}^{\mu+1}}{2 \eta_{2}}=\lambda_{3} \frac{-3 \mathrm{~T}_{3,1}^{\mu+1}+4 \mathrm{~T}_{3,2}^{\mu+1}-\mathrm{T}_{3,3}^{\mu+1}}{2 \eta_{2}} \\
& m=m_{\mathrm{cr}}, m_{\mathrm{cr}}+1, m_{\mathrm{cr}}+2, \ldots
\end{aligned}
$$

Equations (11)-(13) will also be satisfied

for $m=m_{\mathrm{cr}}, m_{\mathrm{cr}}+1, m_{\mathrm{cr}}+2, \ldots$

Here $n_{\tau_{q}}=\frac{\tau_{q}}{\Delta \tau}$, value $m_{\mathrm{cr}}$ is determined by the condition $T_{2,1}^{m_{c r}} \geq T_{\mathrm{cr}}, T_{2,1}^{m_{c r}-1}<T_{\mathrm{cr}}$, and the value $j_{\mathrm{cr}} \quad$ - condition $T_{2, j_{\mathrm{cr}}}^{m} \geq T_{\mathrm{cr}}, T_{2, j_{\mathrm{cr}}+1}^{m}<T_{\mathrm{cr}}$.

Equation (9), (15) is the difference analogue of the differential equations (1), (6) respectively, equations (12), (13) are finite-difference approximation of boundary 
conditions (3), (4), equation (10), (16) is the approximation of the contact conditions on the heat fluxes (5), (7), and (11) is the approximation of the conjugation condition to the temperature (5).

Equations (14) are the nodal values of the temperature at the initial instant of time.

In these approximations, we used a formal replacement by finite-difference secondorder relations of the derivatives with respect to the spatial variable and the first-order finite-difference first-order time derivative in the heat equation, asymmetric finitedifference relations for approximating one-sided derivatives in boundary and contact conditions, so that the difference scheme has approximation order $O\left(h_{1}^{2}+h_{2}^{2}+h_{3}^{2}+\Delta \tau\right)$.

Based on this system of finite-difference equations, the calculation algorithm is written, which is an explicit scheme of the method of finite differences.

It should be noted that the correct choice of steps $\Delta \tau$ and $h_{i}$ when solving a system of finite-difference equations is of great importance. In order to improve the accuracy of calculations, the steps for the coordinate and time should be chosen sufficiently small, however, in order to avoid the instability of the calculations, it is sufficient to satisfy the condition $[10,11]$ :

$$
\Delta \tau \leq \frac{h_{i}^{2}}{2 a_{i}}, i=1,2,3, \Delta \tau \leq \frac{h_{i}^{2}}{2 a_{2}^{g}}
$$

\section{The study results}

The developed algorithm is implemented as a program in the Maple package. It may be noted that the change in temperature at the surface was set experimentally and heated to approximate linear spline time during programming.

We consider the heat transfer in a system whose characteristics are presented in Table 1.

Table 1. Study of the heat transfer process for the metal-PS -metal system.

\begin{tabular}{|c|c|c|c|}
\hline Parameter & The sheet steel I & Styrene styrene & $\begin{array}{c}\text { Smoke - gas-air } \\
\text { mixture }\end{array}$ \\
\hline Thickness, mm & 0.5 & 100 & 100 \\
\hline $\begin{array}{c}\text { Coefficient of thermal } \\
\text { conductivity, } W /(m \cdot K)\end{array}$ & 58 & 0.037 & 0.040 \\
\hline Specific heat, $\mathrm{J} /(\mathrm{kg} \cdot K)$ & 470 & 1340 & 1100 \\
\hline Density, $\mathrm{kg} / \mathrm{m}^{3}$ & 7800 & 35 & 0.7 \\
\hline
\end{tabular}

We assume that the heat transfer coefficient on the heated surface by a fire (hereinafter - medium 1) $\alpha_{1}=60 \mathrm{~W} /\left(\mathrm{m}^{2} \cdot K\right)$, and the coefficient of heat transfer on the surface in contact with the medium without a fire (hereinafter - medium 2) $-\alpha_{2}=3 \mathrm{~W} /\left(\mathrm{m}^{2} \cdot K\right)$.

We assume that the temperature of the medium on the unheated surface is constant in time: $T_{C 2}(\tau)=18^{\circ} \mathrm{C}$. Temperature $T_{\tilde{N} 1}(\tau)$ in the furnace was approximated by a linear $\quad$ spline: $T_{\tilde{N} 1}(\tau)=\frac{\left(\tau_{n}-\tau\right)}{20} T_{\tilde{N} 1}\left(\tau_{n-1}\right)+\frac{\left(\tau-\tau_{n-1}\right)}{20} T_{\tilde{N} 1}\left(\tau_{n}\right), \tau_{n-1} \leq \tau \leq \tau_{n}$, where the temperature values at time instants $\tau_{n}=20 n, n=0,1, \ldots, 15$, are determined experimentally, are presented in the second column of Table 2. 
We accept empirical coefficients $T_{\mathrm{cr}}=60^{\circ} \mathrm{C}, \quad q=1,4 \cdot 10^{5} \frac{\mathrm{W}}{\mathrm{m}^{3}}, \tau_{q}=30^{\circ} \mathrm{C}$ and we assume that the temperature at the internal points of the mixture does not exceed the value at the metal-PS boundary.

The results of calculating the change in the temperature field in the system with time are presented in Table 2.

The upper lines of the table show the values taking into account the heat release during chemical reactions, and in the lower rows, when not accounted for the heat release.

Table 2. Results of calculating the temperature field in the metal-PS -metal system.

\begin{tabular}{|c|c|c|c|c|}
\hline Time, $s$ & $T_{\tilde{N} 1}(\tau)$ & $T_{1}\left(x_{0}, \tau\right)$ & $T_{2}\left(x_{1}, \tau\right)$ & $T_{3}\left(x_{3}, \tau\right)$ \\
\hline 0 & 96 & $\begin{array}{l}18.0 \\
18.0\end{array}$ & $\begin{array}{l}18.0 \\
18.0\end{array}$ & $\begin{array}{l}18.0 \\
18.0\end{array}$ \\
\hline 20 & 158 & $\begin{array}{l}69.8 \\
68.9\end{array}$ & $\begin{array}{l}69.8 \\
69.3\end{array}$ & $\begin{array}{l}18.0 \\
18.0\end{array}$ \\
\hline 40 & 212 & $\begin{array}{l}126.5 \\
121.2\end{array}$ & $\begin{array}{l}126.5 \\
121.2\end{array}$ & $\begin{array}{l}23.7 \\
18.0\end{array}$ \\
\hline 60 & 255 & $\begin{array}{l}179.0 \\
169.2 \\
\end{array}$ & $\begin{array}{l}178.9 \\
169.2\end{array}$ & $\begin{array}{l}35.4 \\
18.0\end{array}$ \\
\hline 80 & 292 & $\begin{array}{l}225.3 \\
212.7 \\
\end{array}$ & $\begin{array}{l}225.3 \\
212.7 \\
\end{array}$ & $\begin{array}{l}50.7 \\
18.0 \\
\end{array}$ \\
\hline 100 & 326 & $\begin{array}{l}266.3 \\
251.8 \\
\end{array}$ & $\begin{array}{l}266.3 \\
251.8 \\
\end{array}$ & $\begin{array}{l}66.9 \\
18.0 \\
\end{array}$ \\
\hline 120 & 347 & $\begin{array}{l}300.5 \\
284.8\end{array}$ & $\begin{array}{l}300.5 \\
284.8 \\
\end{array}$ & $\begin{array}{l}83.5 \\
18.0 \\
\end{array}$ \\
\hline 140 & 371 & $\begin{array}{l}329.2 \\
312.8\end{array}$ & $\begin{array}{l}329.2 \\
312.8 \\
\end{array}$ & $\begin{array}{c}100.2 \\
18.0 \\
\end{array}$ \\
\hline 160 & 381 & $\begin{array}{l}351.9 \\
335.4\end{array}$ & $\begin{array}{l}351.9 \\
335.4\end{array}$ & $\begin{array}{c}116.8 \\
18.0\end{array}$ \\
\hline 180 & 394 & $\begin{array}{l}369.3 \\
353.0\end{array}$ & $\begin{array}{l}369.3 \\
353.0\end{array}$ & $\begin{array}{c}133.0 \\
18.0 \\
\end{array}$ \\
\hline 200 & 401 & $\begin{array}{l}383.0 \\
367.2 \\
\end{array}$ & $\begin{array}{l}383.0 \\
367.2 \\
\end{array}$ & $\begin{array}{c}148.6 \\
18.0 \\
\end{array}$ \\
\hline 220 & 407 & $\begin{array}{l}393.2 \\
378.0\end{array}$ & $\begin{array}{l}393.2 \\
378.0\end{array}$ & $\begin{array}{c}163.6 \\
18.0\end{array}$ \\
\hline
\end{tabular}

As can be seen from Table 2, accounting for chemical reactions greatly affects the temperature change in the system.

Using the obtained model was obtained by solution of the heat problem for the test sample (PS combustion) at $\mathrm{T}_{\mathrm{cr}}=\mathrm{T}_{0}+140^{\circ} \mathrm{C}=18^{\circ} \mathrm{C}+140^{\circ} \mathrm{C}=158^{\circ} \mathrm{C}$, where $\mathrm{T}_{0}=18^{\circ} \mathrm{C}-$ initial temperature. The calculated time for the loss of heat-insulating capacity of the prototype is established, which is 3 minutes 42 seconds.

You can pay attention to the strong acceleration of temperature changes on the surface bordering the surrounding environment. This indicates a strong effect of heat on chemical reactions that occur in PS.

It is also possible to note the practical coincidence of the temperature values at the boundary of the PS -metal and metal-medium, which can be explained by the very small thermal resistance of a thin metal layer with a significant thermal conductivity.

\subsection{Comparative analysis of the results of theoretical and experimental studies of the metal-PS -metal system}


In this division, the calculated and experimental results of studies of the metal-PS - metal multilayered enclosing structures system were compared with those obtained in [12]. For comparative analysis were taken averaged temperature distribution values for the two samples of mark PSW (polystyrene wall). In Fig. 2 shows the time dependence of the temperature change on the external unheated surface of the prototype, based on the results of experimental and theoretical studies.

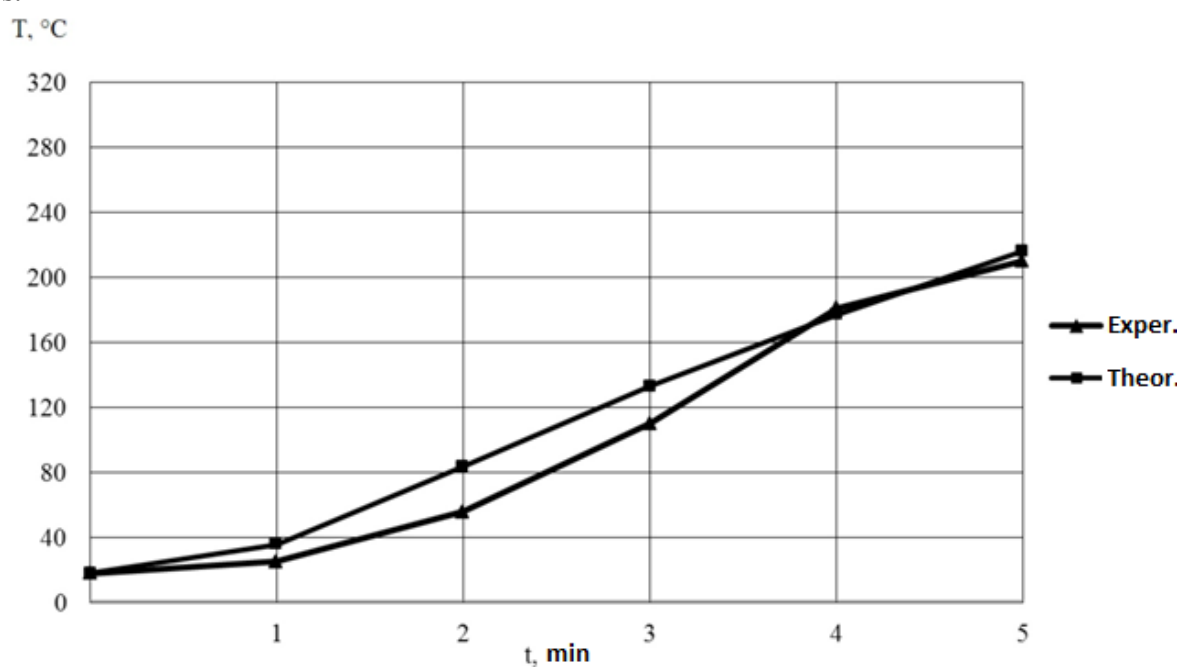

Fig. 2. Comparison of the results of theoretical and experimental studies of prototypes.

As we can see (Fig. 2), the calculated temperature variation curve repeats the experimental curve with small deviations. At the time of the onset of the limit state of fire resistance on the basis of loss of thermal insulating ability, the discrepancy between the temperatures obtained from the results of experimental and theoretical studies is $10^{\circ} \mathrm{C}$, which is an error of $5 \%$, which is acceptable in understanding of practical usage.

\section{Conclusions}

The problem of non-stationary heat transfer process in the test sample and the PSW formed by the quantitative calculation circuit unsteady temperature field in the thickness design scheme based on the explicit finite difference method.

Based on the results of computational and experimental studies, it has been established that the time for the loss of thermal insulation capacity for a prototype of the PSW mark is about 3 minutes 40 seconds. This is a low indicator for fire resistance and does not allow the use of such building structures in accordance with the requirements of regulatory documents of both Ukraine and other states. Obviously, the presence of such structures in buildings can create unfavorable conditions for life and health of people and lead to great material damage in the occurrence and development of a fire. Despite this, it is important to increase the fire resistance of such structures.

\section{References}

1. Yu.V. Polezhaev, F.B. Yurevich, Thermal protection (Moscow: Energy, 1976, In Russian)

2. V.K. Bulgakov, V.N. Kodolov, A.M. Lipanov, Simulation of burning of polymeric materials (Moscow: Chemistry, 1990, In Russian) 
3. P.P. Gyoumdjian, S.V. Kokanin, A.A. Piskunov, Fire and explosion safety 8, 4 (2011) (In Russian)

4. N.M. Belyaev, A.A. Ryadno, Methods of nonstationary heat conductivity (Moscow: Vysh. Shk., 1978, In Russian)

5. I.D. Kashcheyev, Properties and application of refractories. Reference edition (Moscow: Teplotehnik, 2004, In Russian)

6. A.V. Lykov, Theory of heat conductivity (Moscow: Higher Education. Shk. 1967, In Russian)

7. P.P. Shcheglov, V.L. Ivannikov, Fire hazard of polymer materials (Moscow: Stroyizdat, 1992, In Russian)

8. S. Madorskiy, Thermal decomposition of organic polymers (Moscow: The World, 1967, In Russian)

9. V.H. Kodolov, Flammability and fire resistance of polymeric materials (Moscow: Chemistry, 1976, In Russian)

10. Yu.P. Starodub, Mathematical modeling of dynamic seismic problems for the study of the structure of the earth's crust. Direct problem. V.1. - Lviv: V. Stefanyk (Scientific Library of the National Academy of Sciences of Ukraine, 1996, In Ukrainian)

11. Starodub Yu. P. Mathematical modeling of dynamic seismic problems for the study of the structure of the earth's crust. Inverse problem. V.2. - Lviv: V. Stefanyk ( Scientific Library of the National Academy of Sciences of Ukraine, 1996, In Ukrainian)

12. A.P. Polovko, R.B. Veselivsky, O.O. Vasilenko, Yu.Ye. Shelukh, Fire safety: Collection of scientific works. - Lviv: LDU BZD, 19, 118 (2011) (In Ukrainian) 